\title{
Delivering preference for place of death in a specialist palliative care setting
}

\author{
David Oxenham, Anne Finucane, Elizabeth Arnold, Papiya Russell
}

\begin{abstract}
Over the last 10 years, one of the key themes of public policy in palliative care has been achievement of choice in place of death.

In Marie Curie Hospice Edinburgh a baseline audit conducted in 2006 showed that only a small proportion (18\%) of patients referred to hospice services died at home. The audit also revealed that only $31 \%$ of those who expressed a preference to die at home were able to do so, whereas $91 \%$ of those who chose a setting other than home achieved their preference. Overall achievement of preferred place of death was $56 \%$. However a significant number of patients $(29 \%)$ did not have a recorded preference.
\end{abstract}

\begin{abstract}
A programme of quality improvement has continued over the last 7 years to improve identification, communication and achievement of preferred place of death for all patients. The mechanisms to change practice have been: changes to documentation; changes to clinical systems to support use of documentation; support for clinical staff to recognise the value of discussing preferences; and support for clinical staff to develop new skills. In addition the programme has been incorporated into local clinical strategy and this has enabled gaps in service to be addressed with a new service to support early discharge of those patients who wish to die at home.
\end{abstract}

A recent audit showed that all patients had a recorded preference or a documented reason why their preference was unclarified. One third of patients died at home - nearly double the proportion that died at home in the baseline audit. Seventy one per cent of patients who wished to die at home actually died at home - a substantial increase from $31 \%$ at baseline. Achievement of preferred place of death for patients wishing to die in the hospice remained high at $88 \%$.

The focus on assessment of preference for place of death has led to substantial improvements in the identification and achievement of preference for patients dying under the care of the hospice. Furthermore, it has been associated with an increase in the overall proportion of patients who die at home.

\section{Problem}

Palliative care is an approach that improves the quality of life of patients and their families facing the problems associated with lifethreatening illness.[1] Over the last 10 years, one of the key themes of public policy in palliative care has been achievement of choice in place of death. $[2,3]$ A YouGov survey conducted on behalf of Marie Curie Cancer Care in 2005, reported that up to 75 per cent of the general public would choose to die at home; Marie Curie Cancer Care used this and other information in their UK campaign for greater support for people's choice in place of death.

In Marie Curie Hospice Edinburgh, a baseline audit relating to the period November 2005 to March 2006 showed that less than one fifth of patients cared for by the hospice services died at home (30/164 patients). Given the relatively low proportion of home deaths in comparison to the high proportion of preferences for a home death reported in national reports, it became clear that a better understanding of patient preference and improvements in achievement of preference was required. This difference between public campaign and local practice led the hospice senior management team to initiate a programme of quality improvement to improve achievement of patient wishes for place of death.
Three key issues were found to compound the difference. Firstly, existing documentation needed to be improved. The preferred place of death assessment form needed to be reformatted so that more detailed information on patient preferences could be collected and reviewed. Secondly, the challenging nature of assessing place of death preferences meant that some staff avoided initiating discussions about preferred place of death. There was clearly a need to develop the skills of staff in this area. Thirdly, resources needed to be balanced to support dying at home where this was preferred by the patient and family. It was clear that there were sufficient inpatient beds to support a preference to die at the hospice, but not the same resource to support patients who wished to be at home. The lack of rapid access to support at home needed to be addressed.

\section{Background}

To have choice and control over where death occurs is important for a good quality of end of life. $[4,5]$ Consequently, national end of life care strategies recommend that healthcare professionals involve patients approaching end of life, and their carers, in discussions about preferred place of death in order to identity and meet their wishes.[2, 3] 
Much evidence over the last decade has suggested that the majority of the general public would choose home as their preferred place.[6] Findings from studies examining the preferences of patients with advanced illness are mixed, with some reporting that most patients have a home preference $[7,8]$ and others revealing patient preferences for hospice.[9, 10] Given that preferences will vary depending on a range of patient, carer, environmental and informational factors, systems that support discussions about preferences are required, and quality measures that emphasize the proportion of patients dying in their preferred place need to be prioritised.

Open discussion about preferences facilitates anticipatory care planning for patients and helps reduce inappropriate hospital admission at the end of life.[11] Patients who express a preference are less likely to die in hospital compared with those who do not;[10] and are more likely to die at home than in any other location.[12] Congruence between preferred and actual place of death varies greatly with congruence rates (percentage of met preferences for all locations of death) ranging from $30 \%$ to $91 \%$ being reported.[13] Interventions that promote congruence between preferred and actual place of death are required in many settings.

The Preferred Priorities for Care (PPC) tool was developed in 2006 in order to facilitate discussions around end of life care wishes and preferences and to enable communication of care planning and decisions across care contexts (NHS Support Sheet 18). The PPC documentation asks patients to record their preferences and priorities for their future care, and where they would like to be cared for. The document is usually completed by the patient, who then shares it with anyone who is, or likely to be, involved in their care. Recent evaluative studies suggest that this tool is helpful in communicating patient's wishes across care settings.[14] While the PPC is a useful tool, documentation emphasizing continual and proactive review of preferences was deemed more useful for identifying preferences of patients in a specialist palliative care setting.

In this context, Marie Curie Hospice Edinburgh began a programme of improvement to develop systems to support preference identification; and to increase the overall proportion of patients under the care of any hospice service, who die in their place of choice.

\section{Baseline Measurement}

Baseline audit:

All patients referred to Marie Curie Hospice Edinburgh between 1st November 2005 and 7th March 2006 were included from the outset (181 patients). Seventeen patients died before the hospice had contact with them and were excluded, leaving 164 patients.

Of the 164 patients, $70 \%$ died in the hospice, $18 \%$ died at home, $10 \%$ died in hospital and $2 \%$ died in a nursing home.

(Supplementary Material - Table 1)

In terms of preference for place of death, there was no record of any assessment for $29 \%$ of patients. Of the 116 patients who had a recorded preference, $60 \%$ expressed a preference to die at home, $37 \%$ expressed a preference to die at the hospice; $2 \%$ chose a nursing home and $1 \%$ chose a hospital setting. (Supplementary Material - Table 2)

Overall $56 \%$ of patients with a recorded preference died in their preferred place (65/116). Of those who nominated the hospice or care home as their preference, $91 \%$ died in their preferred setting. Only $31 \%$ of those who expressed a preference to die at home actually died at home.

See supplementary file: ds2074.JPG - "Table 1"

\section{Design}

First, the senior management team reviewed and revised the existing documentation relating to preferred place of death (PPoD) assessment. The structure of the original documentation meant that staff only felt able to record a categorical preference - this made it difficult during subsequent review to identify whether a discussion about preferences had happened, and any reasons why it might not have taken place. The documentation did not differentiate between discussions not taking place and situations in which patients had said that they were unsure about preference. Introducing some descriptors of reasons for not discussing PPoD (e.g. 'not known the patient for long enough', or 'it may cause the patient too much distress') reflected the reality of the process of an emerging shared understanding of a plan. Serial recording of 'assessments' reinforced this sense of a continuing process of discussion and planning, reducing the times where there was no recorded preference.

Secondly, the team decided to introduce an expectation of discussing PPoD at each inpatient multidisciplinary team meeting, emphasising the importance of making plans for each patient and enabling clarification when team members held different views or perspectives. Similar processes for review were adopted in the community and day services provided by the hospice. Making the process a routine part of the multidisciplinary meeting also enabled problems to be anticipated and solutions identified.

Next, we emphasised the need to discuss 'preferred place of death' with patients and families rather than 'preferred place of care' as place of death discussions seemed to lead to clearer plans when patients deteriorated. Leadership from senior members of the team was necessary to engage all members of the team in this important difference. Discussions where a patient wanted something that the staff thought was not achievable was initially a challenge. As our team's understanding developed we became more comfortable with the idea that we would not be able to achieve the preference for all our patients. It became possible to share our concerns or problems with the patient and family and arrive at a consensus as each patient's story unfolded.

\section{Strategy}


PDSA cycle 1

Our first aim was to increase the proportion of patients who had a recorded place of death preference; or a reason why preference was not known. The senior management team designed and introduced a structured preferred place of death assessment form. This new form included a section allowing the clinician to select reasons why a preference was not recorded. The form also included space for multiple assessments to point towards a process of regular review. A reaudit six months after the introduction of the new documentation showed that it was still being used inconsistently. Staff had found some of the wording and concepts difficult to use.

\section{PDSA cycle 2}

A need still existed to increase the proportion of patients who had their preferences for place of death recorded, or a clear reason for an unknown preference. Consequently the senior management team further revised the preferred place of death assessment form to improve the visual ease of use and simplify some of the language. The section that listed reasons why preference might not be achieved was removed as this seemed to cause particular confusion. On reaudit, although assessments were carried out more often there were still some patients who did not seem to have had an adequate discussion of their wishes. The senior management team recognised that discussion of patient preferences needed to become embedded into routine clinical assessment.

\section{PDSA cycle 3}

To further increase the proportion of patients with a recorded preference, the senior management team agreed with the service leads (inpatient, community and day services) to introduce regular review of PPoD into the multidisciplinary review meetings, supporting regular MDT discussion and planning. This allowed clinical staff to raise important questions about the purpose of PPoD discussions which in time led to greater buy in from all staff. Discussing patient preferences at the end of life became 'something that we do' and something that is seen to benefit enough patients to make it worthwhile. The proportion of patients with a recorded preference, or a recorded reason why preference was unknown increased. The focus then turned to increasing the proportion of patients dying in their place of choice.

PDSA cycle 4

The development of good assessment and recording of PPoD allowed the senior management team to identify specific issues which prevented patient's from dying in their preferred place. For instance, a preference to die at home was often difficult to achieve when a patient deteriorated more rapidly than expected and a care package could not be arranged quickly enough to enable discharge home. When an opportunity arose to bid for service development money, the senior management team were able to use PPoD audit data to demonstrate that rapid access to health and social care was needed to increase the number of patients able to die in their place of choice. A fast-track discharge service was commissioned and is now used when required. This has contributed to an increase in the proportion of patients wishing to die at home who achieve their wish.

\section{Results}

Reaudit:

One hundred and ninety seven patients died under the care of Marie Curie Hospice Edinburgh during the re-audit period (1 November 2012 to 7 March 2013). One patient died before they were seen by the hospice team leaving 196 eligible patients for the audit. All 196 patients had a PPoD or a documented reason for an unclarified preference.

Of the 196 patients, $51 \%$ died in the hospice, $33 \%$ died at home, $13 \%$ died in hospital; $3 \%$ died in a nursing home and $0.5 \%$ died in a relatives home. ( Supplementary material - Table 3)

Eighty-one per cent of patients had a categorical preference (159 patients) - home was the preferred place for $52 \%$ of patients, hospice was preferred by $43 \%$; while $5 \%$ chose other locations. (Supplementary Material - Table 4). Critically, $71 \%$ of all patients who chose to die at home actually died there - an increase from $31 \%$ during the initial audit in 2005-06. Furthermore, the vast majority of patients who chose to die at the hospice achieved their wish and died there (88\%). (Supplementary Material - Table 5).

Data on reasons for an unclarified preference was also available following the introduction of a more detailed PPoD assessment form. Overall, $19 \%$ of patients (37 patients) had an unclarified preference. The main reasons were that the patient was unable to communicate (11 patients); the patient wasn't known for long enough to have the discussion (10 patients) and the clinician felt that the discussion might cause the patient too much distress (10 patients); other reasons (6 patients).

See supplementary file: ds2075.JPG - "Table 3 "

\section{Lessons and Limitations}

This project shows that it is possible to systematically assess, record and review patient preferences for place of death in a specialist palliative care setting. Following this programme of quality improvement, preference information became available for all patients who died under the care of the hospice. The vast majority of patients $(81 \%)$ had a categorical preference; for the remaining $19 \%$ a clear reason for an unclarified preference was available. The revised documentation also allowed for recording and communication of preference changes; so that all clinicians could see how preferences were evolving for the individual patient over time.

Over the course of this quality improvement programme, the number of patients involved in a discussion about their end of life care preferences increased. Critically, better identification and communication of preferences for patients, in conjunction with the 
introduction of a new fast-track discharge service, contributed to a substantial increase in preference achievement for all patients, in particular those who wanted to die at home. Overall, the number of patients dying at home doubled during the reaudit period compared with the baseline audit. This increase in the number of patients dying at home is potentially cost effective, with recent reports suggesting that the cost of palliative care at home is considerably less than the cost of palliative care in hospital or hospice settings. $[15,16]$

The communication skills of staff also developed over time. The discussion of PPoD at weekly MDT meetings provided an opportunity for more experienced staff members to offer advice and support to staff who sometimes found the initiation of discussions about preferred place of death difficult. Over time staff became more confident in their skills and developed their own communication strategies to sensitively explore the patient's preferences. Such discussions generally took place in context of anticipatory care planning discussions.

Our reaudit data showed that patients choosing to die at home who actually died at home increased to $71 \%$; however patients with a preference to die at home still remain less likely to achieve their wish compared with patients who choose the hospice or a care home. Further increases in preference achievement for home deaths may be difficult unless the structures are in place to support this. Further studies examining the reasons why some patients who choose to die at home end up dying elsewhere, would shed light on the factors that influence the lack of congruence between preferred and actual place of death for this group.

Routine assessment of place of death has enabled more of our patients to engage in discussions about future deterioration and has facilitated better planning and delivery of one important aspect of palliative care. It is not, however, the only measure of quality of a palliative care service. We have not assessed our ability to deliver preference for place of care. It is important that patients are able to die where they wish, but perhaps more important that they are able to live in their preferred setting. Finally, efficiency measures such as reduction in hospital stays (and hospital deaths) should not be conflated with achievement of preference for place of death - a quality measure.

\section{Conclusion}

In 2006 the hospice started to seriously consider the local delivery of national policy on preference for place of death. A Quality Improvement Programme with multiple cycles of PDSA has continued up until 2013. This focus on preference for place of death has been associated with a substantial increase in achievement of preference for patients wishing to die at home (from $31 \%$ to $71 \%$ ). It has also been linked with an increase in the proportion of patients who actually die at home (18\% to $33 \%)$.

\section{References}

1 World Health Organization definition of Palliative Care, 2004. http://www.who.int/cancer/palliative/definition/en/

2 Department of Health. End of Life Care Strategy - Promoting high quality care of all adults at the end of life; 2008.

3 Scottish Government. Living and Dying Well: A national action plan for end of life care in Scotland. Edinburgh; 2008.

4 Smith R. A good death. British Medical Journal. 2000;320:129.

5 Ellershaw J, Dewar S, Murphy D. Achieving a good death for all. British Medical Journal. 2010;341:c4861.

6 Gomes B, Calanzani N, Higginson I. Local preferences and place of death in regions within England: Cicley Saunders International; 2011.

7 Brazil K, Howell D, Bedard M, et al. Preferences for place of care and place of death among informal caregivers of the terminally ill. Palliative Medicine. 2005;19(6):492-9.

8 Higginson I, Sen-Gupta G. Place of care in advanced cancer: a qualitative systematic literature review of patient preferences. Journal of Palliative Medicine. 2000;3:287-300.

9 Gerrard R, Campbell J, Minton O, et al. Achieving the preferred place of care for hospitalized patients at the end of life. Palliative Medicine. 2011;25(4):333-6.

10 Arnold L, Finucane AM, Oxenham D. Preferred place if death for patients referred to a specialist palliative care service. BMJ Supportive \& Palliative Care. 2013; doi: 10.1136/bmjspcare-2012-000338.

11 Munday D, Dale J, Murray S. Choice and place of death: individual preferences, uncertainty, and the availability of care. Journal of the Royal Society of Medicine. 2007;100(5):211-5.

12 Karlsen S, Addington-Hall J. How do cancer patients who die at home differ from those who die elsewhere? Palliative Medicine. 1998 June 1, 1998;12(4):279-86.

13 Bell CL, Somogyi-Zalud E, Masaki KH. Factors Associated with Congruence Between Preferred and Actual Place of Death. Journal of Pain and Symptom Management. 2010;39:591-604.

14 Reed T. How effective is the preferred priorities for care document? NursingTimes. 2011 8th May 2011;107(18).

15 Marie Curie Cancer Care, Understanding the cost of end of life care in different settings, London, Marie Curie Cancer Care, 2012.

16 Sue Ryder, A time and a place: What people want at the end of life. July, 2013.

\section{Declaration of interests}

DO, LA, PBR and AF are employees of Marie Curie Cancer Care, 
an end of life care charity who campaign for choice of place of death and provide end of life care for people in their own homes and in Marie Curie Hospices.

\section{Acknowledgements}

We would like to thank all the staff at Marie Curie Hospice

Edinburgh who have contributed to this team effort. 